\title{
Real-Time Implementation of Greenhouse Monitoring System Based on Wireless Sensor Network
}

\author{
Omar Sedqi Kareem, Noor Najeeb Qaqos
}

\begin{abstract}
The climate change has brought about unpredictable weather conditions that have resulted in the global food shortage being experienced. This issue can be solved by greenhouses, they play a main role in increasing the crop yield per unit area and represent the suitable environment for off-corps yields. Managing and continuous monitoring the green house environment can be done using a wired sensor network, but the high cost, wiring complexity, fixed sensor locations and the restricted distances are the big problems of this type of a networking. To solve these problems, we implemented a real time embedded system using Wireless Sensor Network (WSN) based on ZigBee technology to control and monitor the environmental of greenhouses. The WSN can be adopted as the best solution to apply in greenhouse because of its good properties, long distances, low-cost, low power consumption, high security and high reliability. The constructed system is implemented based on simple components, ATMEGA328P microcontroller and ZigBee are represented the kernel of sensor node, collect data from various sensors and present them to a coordinating station where data can be stored and processed, then actuators will be operate depending on the processed data. The captured data will be displayed for monitoring in a real time manner. The monitor system was developed using GSM technology. The simulation results show that the system is more efficient in the manpower saving and raising the economic value of products. Furthermore, the developed system is simple, and easily installable.
\end{abstract}

Keywords: Greenhouse, Wireless sensor network, ZigBee (WSN), Actuator, Microcontroller.

\section{INTRODUCTION}

Greenhouse systems have been designed for monitoring and controlling microclimatic parameters, like temperature, humidity degree, gas level and light ratio to provide best condition for the plants to grow inside greenhouses [1]. One of the biggest problems for mankind in the 21st century's is food shortage. Global warming and other weather factors have leaded to desertification [2].

The most common used system to control and measurement of environmental parameters in greenhouse depended on wired connection for data transmit. In addition, this traditional system required large numbers of cables for communication and this in turn increase difficulty in the farming of crops, need more effort to installation and maintenance of system, also increasing cost.

Revised Manuscript Received on June 22, 2019.

Omar Sedqi Kareem, Shekhan Technical Institute, Information Technology Department, Duhok Polytechnic University, Duhok, Iraq.

Noor Najeeb Qaqos, Shekhan Technical Institute, Information Technology Department, Duhok Polytechnic University, Duhok, Iraq.
Therefore, high humidity and temperature, also soil containing acid making environmental inside greenhouse invalid, these factor cause shortage of cables' age. The reliability and stability have been affected of the whole system [3]. However, it raises the cost of greenhouse for the advancing control techniques. Moreover, it is difficult for most low education level of farmers to operate on it, thus it is hard to popularize among farmers. So, this leads to a necessity to develop a monitor system with low cost and easy way to get environmental information $[4,5,6,7]$.

B. Basnet, et al. [1], Proposed a prototype design of an automation greenhouse using Arduino microcontroller. with the helpful of LabVIEW program, and analyze acquired signals by the Fast Fourier Transform (FFT). In addition, the system used filter isolated and reduce noise before processing signals by Arduino.

H. Wang, et al. [6], Presented ZigBee as WSN and RS485 bus was used to monitor microclimatic parameters in greenhouse cultivation base. Depending on PC for acquired data and monitoring center for sending command through the coordinator node.

It is better mentioning here that the control greenhouse has importance in reducing effort and cost. Nevertheless, various technologies have been used to measure microclimatic parameters, as mentioned earlier, by using microchip analog devices. This manuscript gives special importance to utilize Global System for Mobile Communications (GSM) with relatively mature and the most commonly used communication system provides with SMS (Short Message Service), voice, data and other business. And along with the development of the communication technology, it is possible to expect for less business expenses. The wireless communication in this paper is based on ZigBee technology and GSM. The environmental parameters will be sent by ZigBee such as carbon dioxide and illumination levels, humidity and temperature degree inside the greenhouse to the users based on their requirements so that users can know the production of greenhouses in time.

This paper is organized as the following; in addition to this introductory section, the section II describes the overall system design. The design of the hardware for the proposed system presents in section III, followed by the design of software in section IV. Results and evaluation of our design introduced in section V. Finally, conclusions are made based on the obtain results in section VI. 


\section{THE OVERALL SYSTEM DESIGN}

The greenhouse automation system is proposed to provide a monitoring and control strategy on the basis of the wireless communication system. A set of nodes sensors have been planted to measure parameters such as temperature and humidity, carbon dioxide concentration and illumination intensity in the greenhouse. To provide real-time information of the greenhouse parameters are transmitted to a coordinator node [8]. The coordinator node calculated the average of the parameters according to data received from different places in greenhouse as shown in Figure 1.

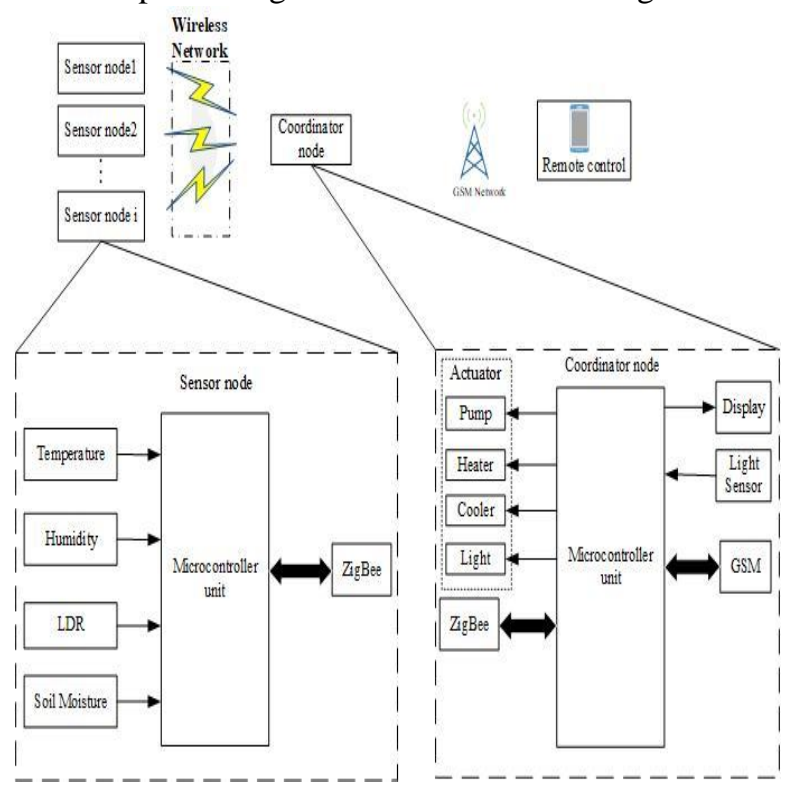

Fig. 1 The schematic diagram of proposed system structure
The purpose of this design is to monitor and control the growing environment due to plants require a limited range of humidity, temperature, light, soil moisture, and air (Nitrogen and Carbon Dioxide, Oxygen) for their growing. Controlling many of these factors will increase plant growth and reproduction.

The communication between the coordinator and the sensors node are a wireless through ZigBee, for remote monitoring and controlling, the coordinator node is contacted with GSM cellular network via a GSM shield.

The most popular environmental parameters can be measured in greenhouses include:

- Heat: - Keeping temperature under control to prevent overheating and freezing, some horticulturalists assume no growth occurs below $\left(10^{\circ} \mathrm{C}\right)$ or above $\left(30^{\circ} \mathrm{C}\right)$.

- Water: - Rainfall isn't directly on soil inside the greenhouse, so the greenhouse depends on irrigation to optimize the controlling water volume and timing for the best growth of plants.

- Light: - When it's too hot shading may be beneficial, to prevent block some of incoming light through covering, also compensation the light using artificial light.

- Humidity and ventilation: - when greenhouse has been totally enclosed, humidity will increase, so growth of plants

\section{Design of Sensor Node}

Sensor nodes in the wireless sensor networks typically contain a number of sensors unit to read parameters in addition to microcontroller unit and the wireless communication unit for data processing and communication, respectively for the overall design such as shown in Figure 2 $[6,7,10]$.

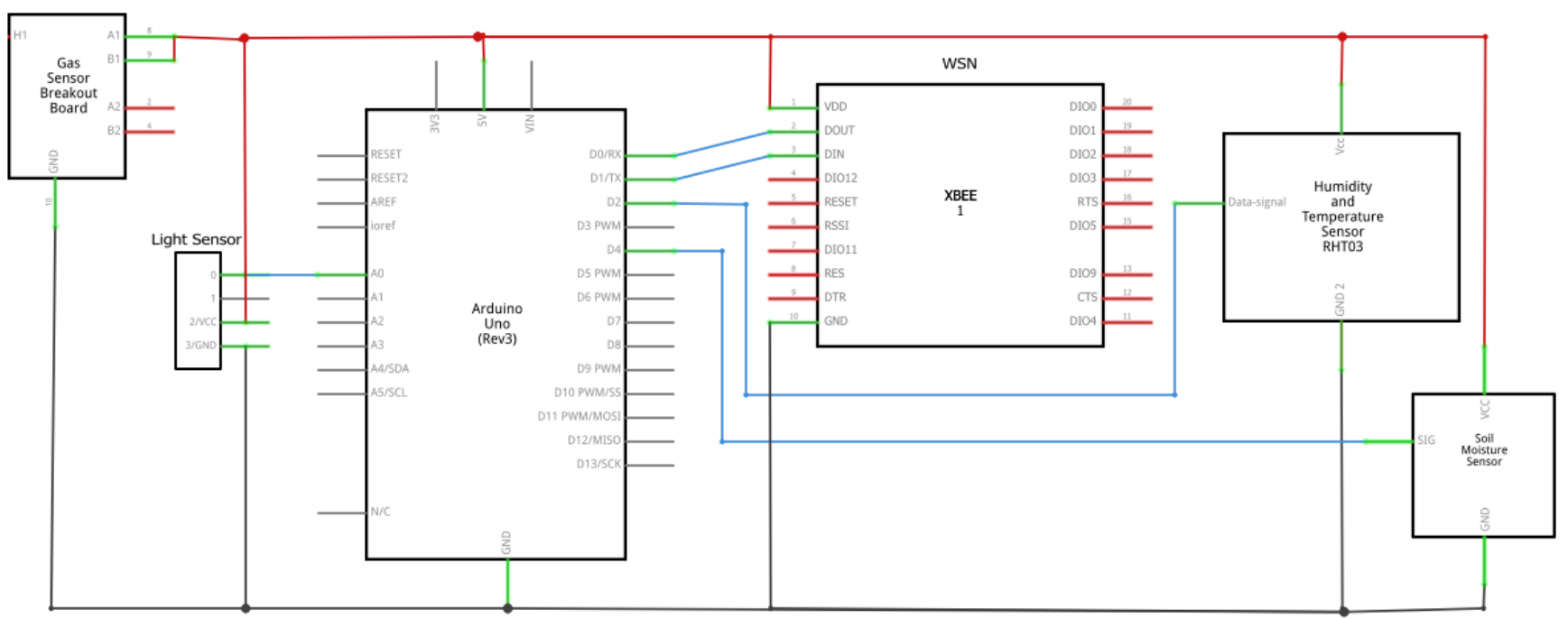

Fig. 2 Implementation of sensor node 
The Arduino MEGA 2560 used in this work because it can achieve all requirements of the system with best performance. It is suitable for collecting and transferring wirelessly the environmental parameters inside greenhouse. The system uses suitable sensors for the greenhouse, the sensors are measuring parameters accurate and can work steadily in the environment of greenhouses. The MEGA 2560 can distinguish the voltage signal, the signal conditioning circuits can transform the current signal to a voltage signal and integrated into the system [10].

\section{Data Acquisition}

The node in WSN represents a type of small embedded device that needs low power consumption and low price. To overcome small space of memory and slow processor capacity, the sensors require to complete data acquisition, transmission and other actions. A sensor node network has been designed to use limited computing and storage to complete the practical application task of the proposed system $[11,12]$.

At this time there are many types of sensors that can be used according to the applications needs. Some factors should be taken in mind in selecting the best type of sensor such as work voltage, power consumption and sampling time. Also, size, price, stability, standard interface, sensitivity and practical life of the sensor must be taken into consideration. Some sensor should be taken in account mainly such as temperature, humidity in greenhouses, air conditioning and light intensity so that the light intensity sensor, temperature sensors, humidity and smoke sensors are chosen based on them.

The sensor nodes are primarily responsible for collecting and transferring data. The air temperature of the greenhouse is determined by the external temperature, the outgoing, incoming radiation, the heating and the cooling system associated with structure of greenhouse and mechanism radiative heat transfer. If one sensor node is used to measure the parameters it must be located in appropriate position, otherwise, if a group of sensors are used, they will be located in different location the results will be better than one node $[13,14]$.

\section{SOFTWARE DESIGN OF THE SYSTEM}

The synchronization of data acquisition and transmission of sensors to the coordinator node is the receiving the command from the network coordinator node and perform the corresponding operation command. The value of each measured environmental variable was estimated based on the readings of the number of sensor nodes using the arithmetic mean over the periods of interest (daytime and night-time for each experimental period), as follow:

$$
\check{x}=\frac{1}{n} \sum_{i=1}^{n} x_{i}
$$

Sensor nodes are connected to the coordinator and transfers the information that are processed by it. The major reason for proposed design is to gather the information in real time, resulting in a lot of data processing, but the sensor nodes are not enough to do the job as shown in Figure 3.

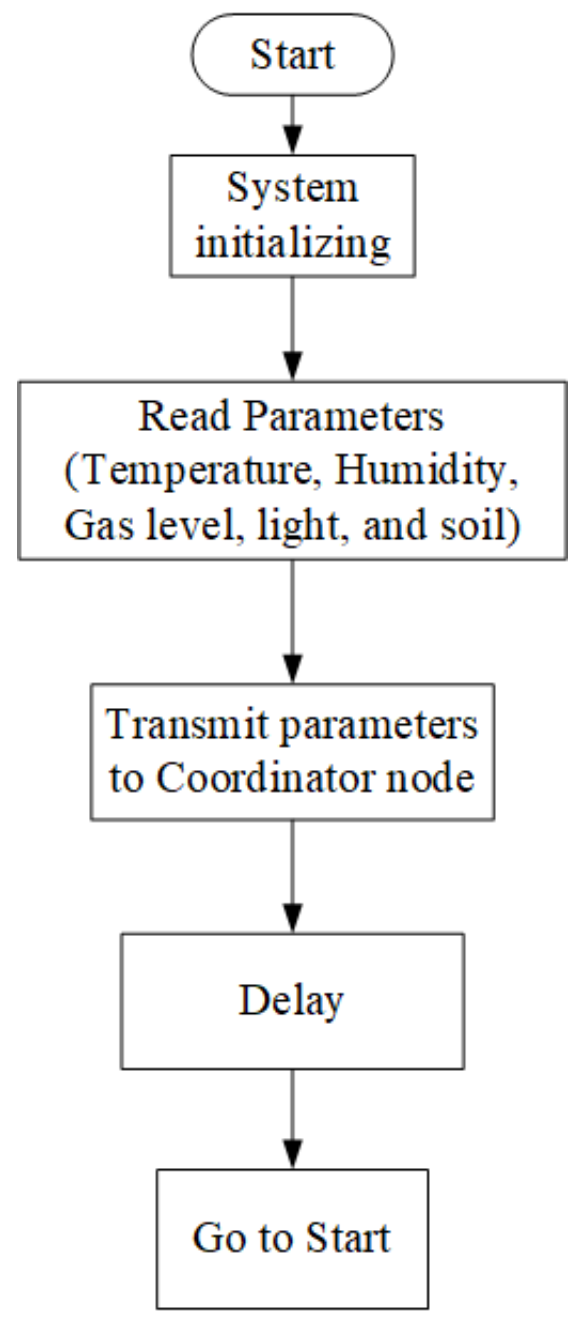

Fig. 3 Flowchart of Sensor node

A coordinated node represents the center of a wireless network of the greenhouse. Its basic function is to regulate wireless networks, communicate with sensors, communicate with the user and verify that the sensor is held normally or not, such as in Figure 4. 


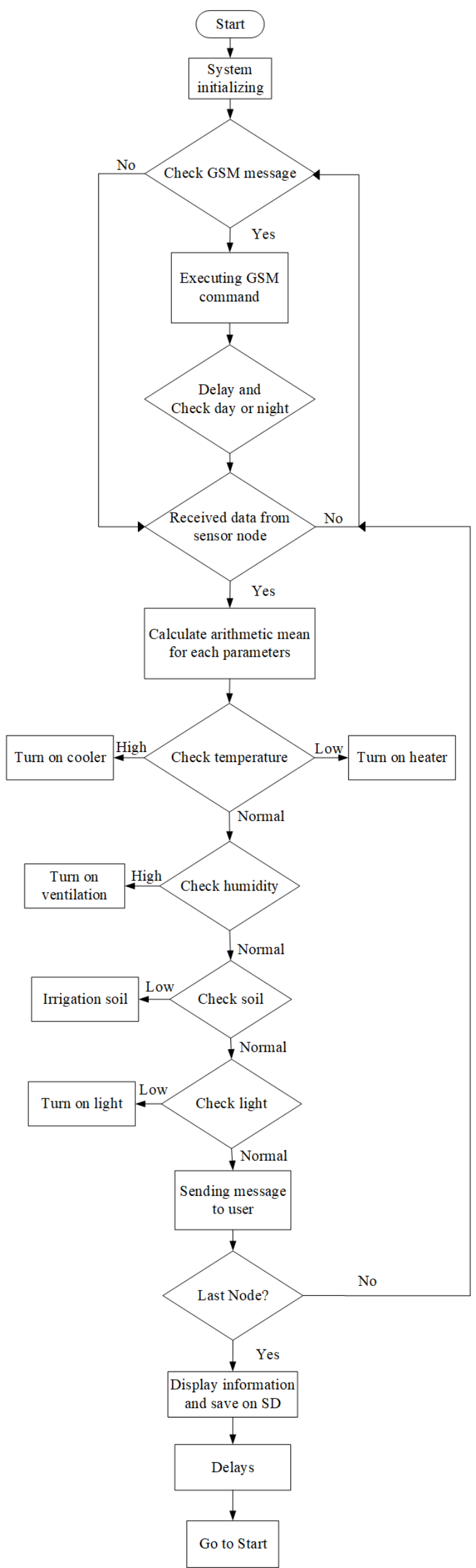

Fig. 4 Flowchart of coordinator node

\section{SYSTEM IMPLEMENTATION AND EVALUATION}

The group of sensors have been uniformly distributed in the greenhouse depending on its size. Also, one coordinator node has been put in to able manage all sensors and control actuators which connected to microcontroller. Under normal conditions, the sensor node will read data from sensors every few minutes, and the node will send real-time data to the coordinator node. When detecting value of parameters more or lower from the standard limit the coordinator will turn on or turn off one of the actuators. The hardware of the proposed system and the status of system, also the average parameters are shown in Figure 5.

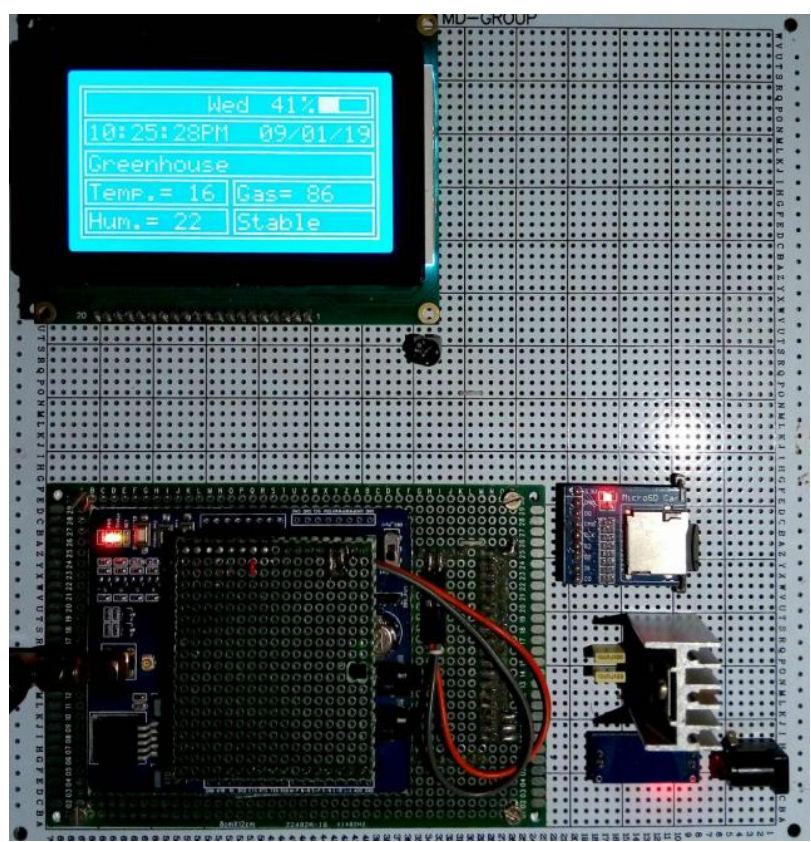

Fig. 5 Implementation of coordinator

The results have been obtained during certain period from testing the system and varying the initial climatic conditions. In the first case, the temperature has been measured during 10 hours, at different two periods one in day and another in night to see the effect of these two periods and increase temperature during day period, as shown in Figure 6.

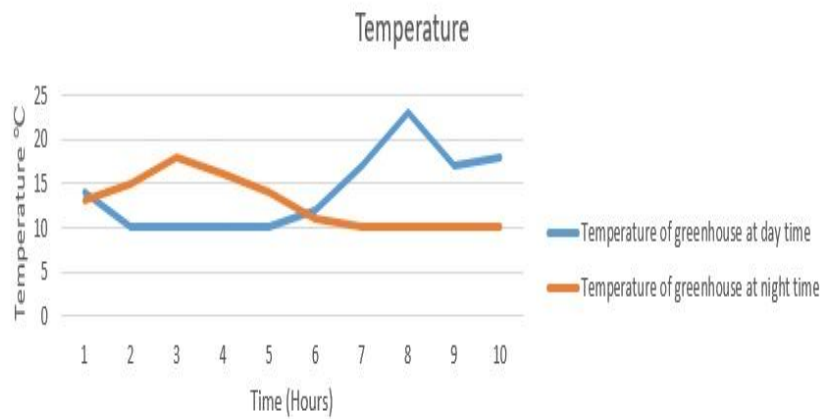

Fig. 6 Measure of temperature during 10 hours.

In the second case, the humidity has been measured also during 10 hours, through the night period has been seen that increasing in humidity appear, as shown in Figure 7. 


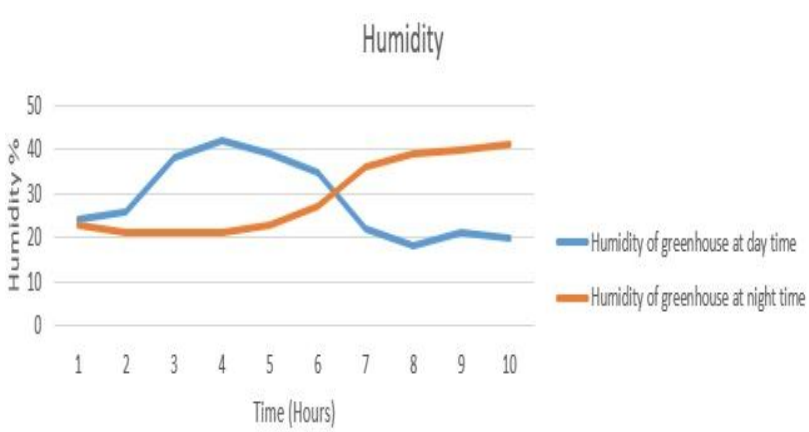

Fig. 7 Measure of humidity during 10 hours

In the third case, the concentration of gas has been measured through the same period, at the night period has been seen that decreasing in the concentration of gas appear, as shown in Figure 8.

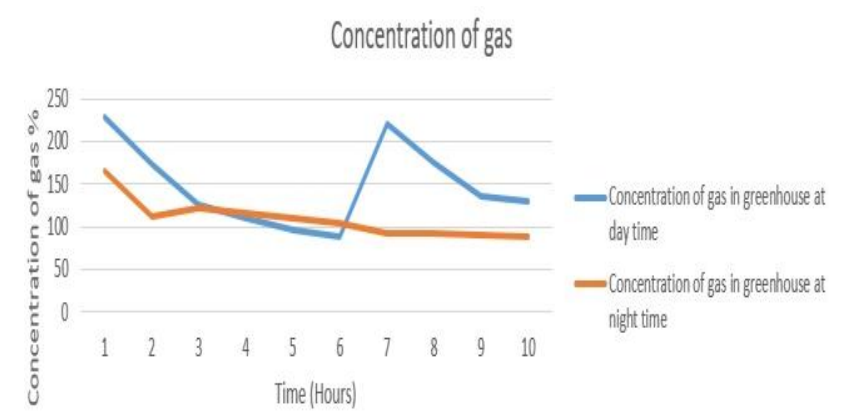

Fig. 8 Measure of gas during 10 hours

\section{CONCLUSIONS}

The proposed design used to control and monitor the different environmental parameters of greenhouses in realtime systems using WSN and ZigBee protocol, parameters include concentration of gas, temperature, light and humidity. This design has many advantages to apply a new technology to the greenhouse for protecting agriculture and avoid using of wire connection systems and give efficient in the manpower saving. Also, supporting large number of nodes which enable expansion in the future easily. The system has been monitored by sending SMS messages to coordinator node. The results appear that the system has good stability, low power consumption, low cost, and efficiently reducing irrigation water consumption, and easy installation and maintenance. The reliability and intelligently for monitoring many environmental factors of greenhouses can be improved by using our proposed design.

\section{ACKNOWLEDGMENT}

This paper has been supported by Duhok Polytechnic University, gratefully acknowledges the generous help for constant guidance and assistance during my work.

\section{REFERENCES}

1. B. Basnet, I. Lee, M. Noh, H. Chun, A. Jaffari, and J. Bang, "A Smart Greenhouse Automation System Applying Moving Average Algorithm", The transactions of The Korean Institute of Electrical Engineers, Vol. 65, No. 10, pp. 1755-1760, November 2016.

2. J. A. Enokela and T. O. Othoigbe, "An Automated Greenhouse Control System Using Arduino Prototyping Platform", Australian Journal of Engineering Research, ISSN: 2203-9465.
3. X. Liu, T. Zhang, B. Li, F. Tian, Y. Tan, Y. Zhang, and H. Lv, "Wireless Measurement and Control System of Environmental Parameters in Greenhouse Based on ZigBee Technology", Proceedings of the 37th Chinese Control Conference July 25-27, 2018, Wuhan, China, pp. 7268-7273.

4. W. Fei-qing, L. Lin-gong, M. Xiu-shui,Q. Jun, and H. Xian-tu, "Development of Wireless Monitor System on Greenhouse Environment Based on GSM", Future Control and Automation, LNEE 172, pp. 371-379, 2012.

5. L. Xiaofeng, Q. Linlin, L. Linjian, and W. Gang, "Design and Implementation of Modern Greenhouse Remote Monitoring System Based on the Android System", Proceedings of the 34th Chinese Control Conference July 28-30, 2015, Hangzhou, China, pp. 5742-5746.

6. H. Wang, X. Dong, Y. Ma, X. Yang, and F. Liu, "Design of Greenhouse Environment Wireless Monitoring System Based on ZigBee", Informatics in Control, Automation and Robotics, LNEE 132, pp. 579-586, 2011.

7. L. Xia, L. Wenhui, S. Yixin, "Greenhouse Monitoring System Design Based on MSP430 and King view", IEEE, 978-1-5386-2901-7/17, pp. 111-114, 2017.

8. L. Bajer and O. Krejcar, "Design and Realization of Low-Cost Control for Greenhouse Environment with Remote Control", IFAC (International Federation of Automatic Control) Hosting by Elsevier Ltd., pp. 368-373, 2015 .

9. J. Both, L. Benjamin, J. Franklin, G. Holroyd, L. D. Incoll, M. G. Lefsrud, and G. Pitkin, "Guidelines for measuring and reporting environmental parameters for experiments in greenhouses", NCERA-101, Department of Environmental Sciences, Rutgers University, Newark, USA, DOI 10.1186/s13007-015-0083-5, 2015.

10. R. Algarín, J. C. Cabarcas, and A. P. Llanos, "Low-Cost Fuzzy Logic Control for Greenhouse Environments with Web Monitoring", Electronics, 6, 71; doi:10.3390/electronics6040071, 23 September 2017.

11. Y. Liu and C. Bi, "The Design of Greenhouse Monitoring System based on ZigBee WSNs", IEEE International Conference on Computational Science and Engineering (CSE) and IEEE International Conference on Embedded and Ubiquitous Computing (EUC), pp. 430433, 2017.

12. A.Lambebo and S. Haghani, "A Wireless Sensor Network for Environmental Monitoring of Greenhouse Gases", ASEE 2014 Zone I Conference, April 3-5, 2014, University of Bridgeport, Bridgpeort, CT, USA.

13. K. P. Ferentinos, N. Katsoulas, A. Tzounis, T. Bartzanas, and C. Kittas, "Wireless sensor networks for greenhouse climate and plant condition assessment", IAgrE. Published by Elsevier Ltd., pp. 70-81, 14 November 2016.

14. X. Li, X. Cheng, K. Yan, and Peng Gong, "A Monitoring System for Vegetable Greenhouses based on a Wireless Sensor Network", Sensors 2010, 10, 8963-8980; doi:10.3390/s101008963. 